\title{
Galactic Halos as Boson Stars
}

\author{
Jae-weon Lee and In-gyu Koh \\ Department of Physics, \\ Korea Advanced Institute of Science and Technology, \\ 373-1, Kusung-dong, Yusung-ku, Taejeon, Korea
}

\begin{abstract}
We investigate the boson star with the self-interacting scalar field as a model of galactic halos. The model has slightly increasing rotation curves and allows wider ranges of the $\operatorname{mass}(m)$ and $\operatorname{coupling}(\lambda)$ of the halo dark matter particle than the non-interacting model previously suggested(ref.[3]). Two quantities are related by $\lambda^{\frac{1}{2}}\left(m_{p} / m\right)^{2} \gtrsim 10^{50}$.
\end{abstract}


It is well known that the flatness of the galactic rotation curves indicates the presence of dark matter around galactic halos. However, the properties of the dark matter are still mysterious. For example, why the dark matter in halos does not fall towards the center of galaxy and form black holes? The answer to the above question may be a good criterion for the good halo model.

There are thermal distribution model [1] where density profile $\rho \sim r^{-2}$, and spherical infall model[2] where $\rho \sim r^{-2.25}$.

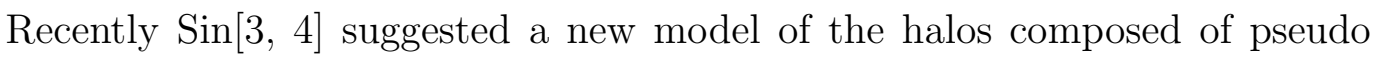
Nambu-Goldstone boson (PNGB).

According to the model, the condensation of ultra light PNGB whose Compton wavelength $\lambda_{\text {comp }}=\frac{\hbar}{m c}$ is about $R_{\text {halo }}$ is responsible for the halo formation. Cosmological role of the ultra light PNGB was studied in the late time phase transition model [5] to reconcile the smoothness in the background radiation with the large scale structure.

Before Sin's work, an astronomical object which consists of the PNGB dark matter was suggested by some authors [6]. In their model the force against gravitational collapse comes from the momentum uncertainty of the quantum mechanical uncertainty principle.

Since the typical length scale $R$ in this model is Compton wavelength $\lambda_{\text {comp }} \sim$ $\frac{1}{m}$ of the particle, the typical mass scale of the object is $M \sim \frac{R}{G} \sim \frac{m_{p}^{2}}{m}$.

Similarly, in Sin's model galactic halos are the objects of the self-gravitating 
bose liquid whose collapse are prevented by the uncertainty principle.

The typical halo has radius $R_{\text {halo }} \sim 100 \mathrm{kpc} \sim 10^{24} \mathrm{~cm}$ and mass $M_{\text {halo }} \sim$ $10^{12} M_{\odot} \sim 10^{45} \mathrm{~g}$, so one find the mass $m$ of the PNGB whose de Broglie wave length $\sim R_{\text {halo }}$ is about $10^{-26} \mathrm{eV}$.

Note that the de Broglie length $\sim \frac{c}{v} \times \lambda_{\text {comp }}$ is more adequate to our purpose.

The self-gravitating condensed states are described by the following non-linear Schröedinger equation:

$$
i \hbar \partial_{t} \psi=-\frac{\hbar^{2}}{2 m} \nabla^{2} \psi+G m M_{0} \int_{0}^{r^{\prime}} d r^{\prime} \frac{1}{r^{\prime 2}} \int_{0}^{r} d r^{\prime \prime} 4 \pi r^{\prime \prime 2}|\psi|^{2} \psi(r),
$$

which was known as the Newtonian limit of the boson star fields equation[7].

The normalization constant $M_{0}$ is chosen to give the total mass of halo $M=$ $M_{0} \int d r 4 \pi r^{2}|\psi|^{2}$ as in ref.[3].

The rotation velocity of the stellar object rounding halo at radius $r$ is given by

$$
V(r)=\sqrt{\frac{G M(r)}{r}},
$$

where $M(r)$ is mass within $r$.

Integrating eq.(11) numerically and using eq.(2) Sin found slightly increasing rotation curves and density profile $\rho \sim r^{-1.6}$.

What happens if there are repulsive self-interactions between the dark matter particles? To answer this and stability question it is desirable to study the relativistic fields equations than the Schröedinger equation. 
The cold gravitational equilibrium configurations of massive scalar field were found by solving the Klein-Gordon equations with gravity decades ago [8. We find that these configurations, called boson star [9], are adequate to the relativistic extension of Sin's model.

Consider a self-interacting complex scalar field and the gravity whose action is given by

$$
S=\int \sqrt{-g} d^{4} x\left[\frac{-R}{16 \pi G}-\frac{g^{\mu \nu}}{2} \phi_{; \mu}^{*} \phi_{; \nu}-\frac{m^{2}}{2}|\phi|^{2}-\frac{\lambda}{4}|\phi|^{4}\right] .
$$

Since halos seem to be spherical, we choose Schwarzschild metric

$$
d s^{2}=-B(r) d t^{2}+A(r) d r^{2}+r^{2} d \Omega
$$

and assume spherically symmetric field solutions

$$
\phi(r, t)=(4 \pi G)^{-\frac{1}{2}} \sigma(r) e^{-i \omega t} .
$$

From the action, dimensionless time independent Einstein and scalar wave equations appear as in ref.[10]:

$$
\begin{gathered}
\frac{A^{\prime}}{A^{2} x}+\frac{1}{x^{2}}\left[1-\frac{1}{A}\right]=\left[\frac{\Omega^{2}}{B}+1\right] \sigma^{2}+\frac{\Lambda}{2} \sigma^{4}+\frac{\sigma^{\prime 2}}{A}, \\
\frac{B^{\prime}}{A B x}-\frac{1}{x^{2}}\left[1-\frac{1}{A}\right]=\left[\frac{\Omega^{2}}{B}-1\right] \sigma^{2}-\frac{\Lambda}{2} \sigma^{4}+\frac{\sigma^{\prime 2}}{A}, \\
\sigma^{\prime \prime}+\left[\frac{2}{x}+\frac{B^{\prime}}{2 B}-\frac{A^{\prime}}{2 A}\right] \sigma^{\prime}+A\left[\left(\frac{\Omega^{2}}{B}-1\right) \sigma-\Lambda \sigma^{3}\right]=0,
\end{gathered}
$$

where $x=m r, \Omega=\frac{\omega}{m}, A \equiv\left[1-2 \frac{M(x)}{x}\right]^{-1}$ and $\Lambda=\frac{\lambda m_{p}^{2}}{4 \pi m^{2}}$.

One may take $M(x)$ for dimensionless mass of the boson star for large $x$. 
Numerical solutions of the above equations are studied by many authors 11 , 12, 13. The required boundary conditions are $M(0)=0, \sigma^{\prime}(0)=0$ and $B(\infty)=$ 1 and free parameters are $\sigma(0)$ and $\Omega$.

For the case $\Lambda=0$ [12 it was found that there is a maximum mass $M_{\max }=$ $0.633 \frac{m_{p}^{2}}{m}$ for the zero node solution.

We will focus on the non-zero node solutions, because the rotation curve of the zero node solution falls too fast to explain the flatness of the rotation curves of many galaxies(see ref. 四 for more arguments). This raises the stability problem of higher node solutions, which will be discussed later.

Maximum masses for higher node solutions are proportional to node number $n$ and about the same order as for the zero node case for small $n$.

This with $M_{\text {halo }}$ gives us $m \lesssim 10^{-22} \mathrm{eV}$.

Another constraint comes from the maximum stable center density against small radial perturbation [13] $\rho_{c}=2.1 \times 10^{98} \mathrm{~m}^{2} \mathrm{~g} / \mathrm{cm}^{3}>10^{-24} \mathrm{~g} / \mathrm{cm}^{3}$, which is equivalent to $m \gtrsim 10^{-28} \mathrm{eV}$ for the zero node solutions. So for the zero node solutions $10^{-28} \mathrm{eV} \lesssim m \lesssim 10^{-22} \mathrm{eV}$

For the case $\Lambda \neq 0$, new scale appears because of the repulsive force preventing halo from gravitational collapse. In this case the typical length scale is $R \sim \Lambda^{\frac{1}{2}} / m$, thus the typical mass scale is $\frac{R}{G} \sim \Lambda^{\frac{1}{2}} m_{p}^{2} / m$, which is also of order maximum mass like $\Lambda=0$ case.

Numerical study shows $M_{\max }=0.22 \Lambda^{\frac{1}{2}} \frac{m_{p}^{2}}{m}$ for zero node solutions. From 
the fact that $M_{\max }>M_{\text {halo }}$ we find

$$
\lambda^{\frac{1}{2}}\left(\frac{m_{p}}{m}\right)^{2} \gtrsim 10^{50}
$$

This is a relation between the mass and coupling of the halo dark matter particle. For the perturbative case $(\lambda<O(1))$ the above relation implies $m \lesssim 10^{3} \mathrm{eV}$.

To treat particles as a classical field, we require that the inter-particle distance should be smaller than their Compton wave length. This gives $m \lesssim 10^{-2} \mathrm{eV}$.

Note that $\Lambda=\lambda m_{p}^{2} / 4 \pi m^{2}$ is very large even for very small $\lambda$ due to the smallness of $m$ relative to $m_{p}$, hence the self-interaction effect is non-negligible.

Were there any realistic particle physics model satisfying the above relation? Unfortunately, the usual cosine potential $V(\phi)=\mu^{4}(1-\operatorname{Cos}(\phi / f))$ for the PNGB is inappropriate for our study, because the sign of the quartic coupling constant is negative in the Taylor expansion about the potential minima and $\phi$ is real. Real scalar field such as axion may form oscillating soliton star[14] rather than the boson star.

Instead, we consider the following potential.

$$
V(\phi)=\mu^{4}\left(1+\left(\frac{\phi}{f}\right)^{2}\right)^{2}
$$

Inserting mass and quartic coupling from the above potential to the relation in eq.(9), we get $0.1\left(\frac{m_{p}}{\mu}\right)^{2} \gtrsim 10^{50}$ and equivalently $\mu \lesssim 10^{2} \mathrm{eV}$.

We also solve the equations numerically and find the dimensionless rotation velocity which is given by $V_{\text {rot }}=\sqrt{M(x) / x}=\left[\frac{1}{2}\left(1-A^{-1}\right)\right]^{\frac{1}{2}}$. 
The results are shown in fig.1 and fig. 2 .

Fig.1 shows rotation velocity curves for the cases $\Lambda=0$ and $\Lambda=300$. The parameters are $B(0)=0.641, \sigma(0)=0.1$ and $B(0)=0.781, \sigma(0)=0.01$ respectively.

Fig. 2 shows $\sigma$ and rotation velocity curve of 8 nodes $\operatorname{solution}(n=9)$.

It is interesting that the line connecting minimum points of the rotation velocity is almost straight. For large $n$ and $\Lambda \gg 1$ mass profile is $\rho \sim r^{-1.7}$. Rotation curves are slightly increasing regardless of the self-interactions.

Including the visible matter may change the slope of the curves and explain the variety of the observed galaxy rotation curves as shown in ref. [4.

We will now study the Newtonian limit of our model. The strength of the gravity of halo $G M_{\text {halo }} / R_{\text {halo }}$ is comparable to that of the earth. Therefore we can use the Newtonian limit $\Omega=\frac{\omega}{m}=\sqrt{1+\left(\frac{k}{m}\right)^{2}} \rightarrow 1$, which is comparable to the Newtonian gravity approximation $2 M(x) / x \ll 1$. Collecting terms to $O\left(\xi^{2}=\left(\frac{k}{m}\right)^{2}\right)$ one can find that for $\Lambda=0$ the equations of motion are 15

$$
\begin{gathered}
\nabla^{2} \sigma=\gamma \sigma \\
\nabla^{2} \gamma=2 \sigma^{2},
\end{gathered}
$$

where $\gamma \equiv 1-\frac{\Omega^{2}}{B}$.

Integrating eq.(12) and inserting the result into eq.(11) we can find

$$
\frac{1}{2} \nabla^{2} \sigma=\left(E+\int_{0}^{x} d x^{\prime} \frac{1}{x^{\prime 2}} \int_{0}^{x^{\prime}} d x^{\prime \prime} x^{\prime \prime 2} \sigma^{2}\right) \sigma
$$


which is a dimensionless version of eq.(四). Here $E$ is a integral constant.

Therefore one may treat the Bose liquid model as a Boson star model.

It is useful to study the scaling properties of eq.(11) and eq.(12) for analyzing numerical solutions.

Rescaling the total number of charges $N=Q \propto \int \sigma^{2} x^{2} d x l$ times increases the mass $l$ times.

Equations (11) and (12) are invariant under this rescaling when

$$
x \rightarrow l^{-1} x, \sigma \rightarrow l^{2} \sigma, \gamma \rightarrow l^{2} \gamma
$$

which is consistent with the model in ref.[3].

So one may say that for the non-interacting case the heavier halos are smaller in size.

It is difficult to find the scaling properties for the case $\Lambda \neq 0$.

For the case $\Lambda \gg 1$ further rescaling : $\sigma_{*}=\sigma \Lambda^{1 / 2}, x_{*}=x \Lambda^{-1 / 2}$ and $M_{*}=$ $M \Lambda^{-1 / 2}$ with neglecting terms to $O\left(\Lambda^{-1}\right)$ yields wave equations:

$$
\begin{gathered}
\sigma_{*}^{2}=\left(\Omega^{2} / B-1\right)=-\gamma, \\
M_{*}^{\prime}=\frac{1}{4} x_{*}^{2}\left(3 \Omega^{2} / B+1\right)\left(\Omega^{2} / B-1\right), \\
\frac{B^{\prime}}{A B x_{*}}-\frac{1}{x_{*}^{2}}\left(1-A^{-1}\right)=\frac{1}{2}\left(\Omega^{2} / B-1\right)^{2},
\end{gathered}
$$

which are also shown in ref. 10 .

Following the same procedure of $\Lambda=0$ case, we get the Newtonian limits of eq.(16) and eq.(17). 


$$
\nabla^{2} \gamma=2 \sigma_{*}^{2}=-2 \gamma
$$

whose solutions are

$$
\gamma=-\gamma_{0} \frac{\operatorname{Sin}\left(\sqrt{2} x_{*}\right)}{\sqrt{2} x_{*}}
$$

and

$$
\sigma_{*}=\sqrt{\frac{\gamma_{0} \operatorname{Sin}\left(\sqrt{2} x_{*}\right)}{\sqrt{2} x_{*}}},
$$

where $\gamma_{0}=|\gamma(0)|$.

The above approximation is invalid when $x_{*}$ is large and $n>1$.

As expected, the typical length scale is $m^{-1} \Lambda^{\frac{1}{2}}$. These solution do not show simple scaling property, however numerical study indicates that when the central density is lesser than the critical value corresponding to $M_{\max }$, heavier halo has smaller radius for both $\Lambda=0$ and $\Lambda \neq 0$ cases. [11]

Note the facts that the above arguments are valid when the node number is fixed and both mass and radius of boson star are increasing function of the node number.

Now, let us discuss the stability of higher node solutions. There are studies 12 , 13] indicating that non-zero node solutions with $\Lambda=0$ are unstable against fission and the small radial perturbation.

Since higher node solutions are unstable, they must be long-lived to explain the age of galaxies. 
One possible decay mechanism for non-zero node solution to zero node solution is gravitaional radiation 18 . The power of gravitaional radiation $P$ is about $G\left(d^{3} I / d t^{3}\right)^{2}$, where the quadrapole moment $I \sim M_{\text {halo }} R_{\text {halo }}^{2}$. The available time scale is $T \sim\left(R_{\text {halo }}^{3} / G M_{\text {halo }}\right)^{1 / 2}$, which is given by the virial theorem. The parameter $\alpha \equiv G M_{\text {halo }} / R_{\text {halo }}$ indicates how much halo is relativistic.

So we can find a crude estimate of power $P \sim G^{4}\left(M_{\text {halo }} / R_{\text {halo }}\right)^{5} \sim \alpha^{5} / G \sim$ $\alpha^{5} 10^{59} \mathrm{ergs} / \mathrm{s}$. Since $\alpha \sim 10^{-7}$ for halo, $P \sim 10^{24} \mathrm{erg} / \mathrm{s}$. The potential energy of halo $G M_{\text {halo }}^{2} / R_{\text {halo }}$ is about $10^{58} \mathrm{erg}$, and therefore time scale of the decay by the gravitational radiation is much longer than the age of galaxies $\sim 10^{10} \mathrm{yr} \sim 10^{17} \mathrm{~s}$.

For the $\Lambda \neq 0$ case, there is a work indicating that higher node solutions are stable against the perturbation with fixed particle number [19. However, there seems to be no work on the stability against the more general perturbations. Since it is still unclear that higher node solution with $\Lambda \neq 0$ is stable, we must again estimate the life time of halo against gravitational radiation. From eq.(15) we get $\Lambda \sigma^{4}=-\gamma \sigma^{2}$, which indicates that in Newtonian limit the energy of the repulsive force is comparable to the gravitaional potential energy. So the energy distribution in halo is not so different from the $\Lambda=0$ case. Therefore, we argue that the same procedure for calculating the gravitaional radiation is applicable to the $\Lambda \neq=0$ case and halo is long-lived against the gravitaional radiation.

Another cooling mechanism, evaporation and collapse procedure, is also inefficient [3].

In conclusion, we find that self-interactions between the particles, even weak, 
may play important role in the boson star model of halos.

Our work can be easily extended to the Boson-Fermion star 16 and Q-star 17.

\section{ACKNOWLEDGMENTS}

This work was supported in part by KOSEF. One of authors(Lee) are thankful to M. Gleiser, P. Jetzer and A. Liddle for helpful comments. 


\section{References}

[1] D. Lynden-Bell, MNRAS 136, 101 (1967), I. King Astron. J., 71, 64 (1966)

[2] J. Gott, Ap. J. 201 , 296 J. Gunn, Ap. J. 218 , 592 J. Fillmore and P. Goldreich, Ap. J. 281, 1

[3] S.J. Sin, Phys. Rev., D50, 3650(1995)

[4] S.U. Ji and S.J. Sin,Phys. Rev., D50, 3655(1995)

[5] C. W. Hill, D. N. Schramm and J. N. Fry ,Comments. Nucl. Part. Phys., $19,25(1989)$

[6] W. H. Press and B. S. Ryden ,Phys. Rev. Lett., 64, 1084 (1990)

[7] For example, see E. Seidel and W. Suen, ,Phys. Rev., D42, 384 (1990)

[8] D.J. Kaup, Phys. Rev., 172,1331 (1968), R. Ruffini and Bonazzola, Phys. Rev. 137, $1767(1969)$

[9] For a review see P. Jetzer,Phys. Rep., 220, 163 (1992), T.D. Lee and Y. Pang, Phys. Rep., 221, 251 (1992) ,A. Liddle and M. Madsen, Int.J.Mod.Phys., D1 ,101 (1992) and references there in.

[10] M. Colpi and S.L. Shapiro and Ira Wasserman, Phys. Rev. Lett., 57, 2485 $(1986)$

[11] P. Jetzer and V. Bij, Phys. Lett., B227, 341 (1989) 
[12] R. Friedberg, T. D. Lee and Y. Pang, Phys. Rev., D35, 3640 (1987)

[13] M. Gleiser, Phys. Rev., D38 , 2376 (1988), P. Jetzer, Nucl. Phys., B316 , 411 (1989), M. Gleiser and R. Watkins, Nucl. Phys., B319 , 733 (1989)

[14] E. Seidel and W. Suen, Phys. Rev. Lett., 66 , 1659 (1991)

[15] T. D. Lee and Y. Pang, Nucl. Phys., B315,477 (1989)

[16] A. Henriques, A. Liddle and R. Moorhouse Phys. Lett., B233, 99 (1989), ; Nucl. Phys., B337, 737 (1990)

[17] B. W. Lynn, Nucl. Phys., B321 , 465 (1989)

[18] R. Ferrell and M. Gleiser, Phys. Rev., D40 , 2524 (1989)

[19] Ph. Jetzer, Phys. Lett., B222, 447 (1989) 


\section{Figure Captions}

\section{Figure 1}

Rotation velocities as a function of rescaled $x$ for the parameters $\Lambda=0$ (thin line) and $\Lambda=300$ (thick line). $\Omega$ is 0.9 . The real values of the $x$ end are 80 and 220 respectively.

\section{Figure 2}

Rotation velocity and $10 \times \sigma$ as a function of position $x$ for $n=9$ solution. The parameters are $\Lambda=300, \Omega=0.9, B(0)=0.780$ and $\sigma(0)=0.01$. 
Figure 1:

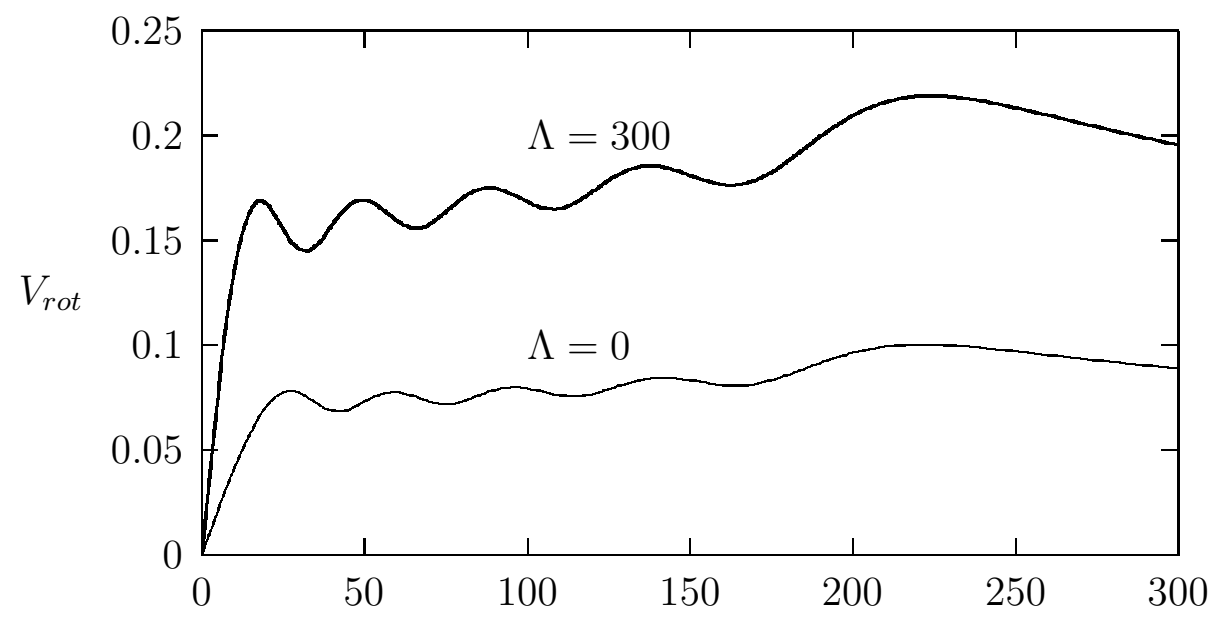

Figure 2:

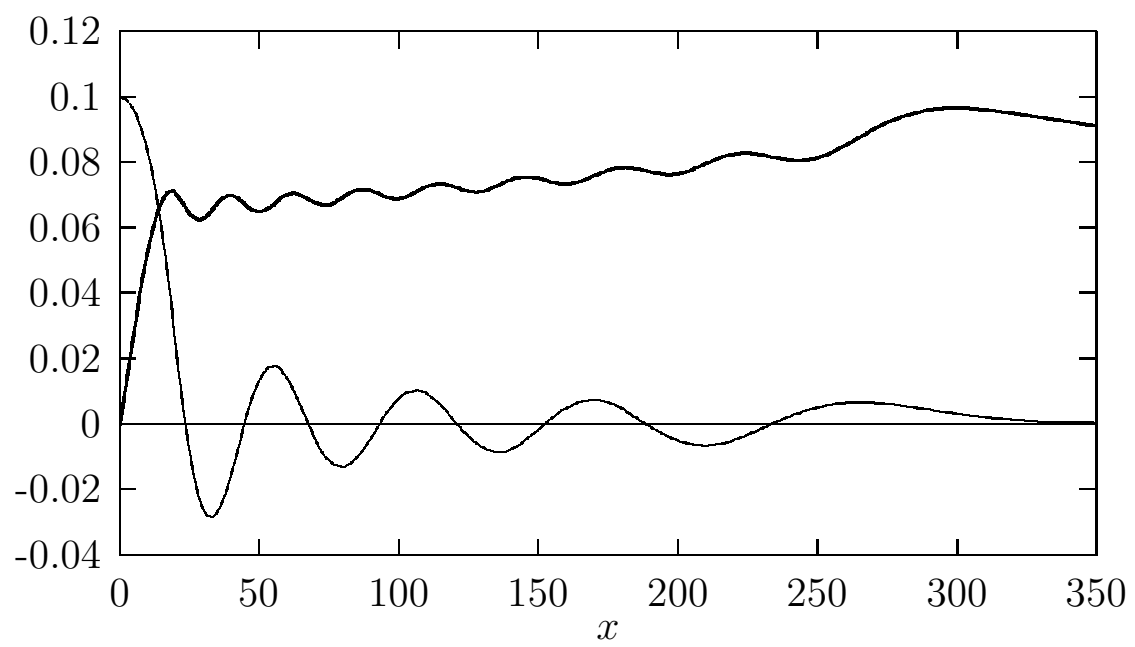

